\title{
Prevalencia de infección del tracto urinario y perfil de susceptibilidad antimicrobiana en Enterobacterias
}

\section{Prevalence of urinary tract infection and antimicrobial susceptibility profile in Enterobacteriaceae}

\section{Prevalência de infecção do trato urinário e perfil de susceptibilidade antimicrobiana em Enterobacteriaceae}

\author{
María Gabriela Carriel Álvarez \\ gcarriela@gmail.com \\ https://orcid.org/0000-0002-8917-7552
}

Jonnathan Gerardo Ortiz

jonnathan.ortiz@ucacue.edu.ec

https://orcid.org/0000-0001-6770-2144

\section{Programa de Maestría en Diagnóstico de Laboratorio Clínico y Molecular, Universidad Católica de Cuenca-Ecuador}

Recibido 26 de febrero 2021 | Arbitrado y aceptado 19 de marzo 2021 | Publicado en 4 de mayo 2021

\begin{abstract}
RESUMEN
Las Infecciones del Tracto Urinario (ITU), constituyen uno de los principales motivos de consulta en el ámbito de atención primaria, debido al aumento de la resistencia antibacteriana. Objetivo. Caracterizar la prevalencia de infección del tracto urinario y el perfil de susceptibilidad antimicrobiana in vitro en Enterobacterias en los pacientes de la provincia de Santa Elena Ecuador. Método. Esta investigación fue descriptiva de diseño documental. La población fue de 827 registros de urocultivos, recopilados de la base de datos del laboratorio de microbiología del Centro de Especialidades IESS La Libertad, en el período comprendido desde agosto 2019 hasta marzo de 2020. Los datos fueron procesados mediante estadística descriptiva, análisis de frecuencia y chi cuadrado. Resultados. De este estudio indican que la prevalencia de ITU fue 22,1\%; los principales agentes etiológicos fueron: E. coli $(76,0 \%)$, Klebsiella oxytoca $(6,5 \%)$, Klebsiella pneumoniae $(5,8 \%)$ y Proteus mirabilis $(3,9 \%)$. La ITU y la infección por E. coli fueron estadísticamente mayores en mujeres y adultos mayores. La mayor frecuencia de resistencia de E. coli fue para ácido nalidíxico $(81,2 \%)$, ampicilina $(79,9 \%)$, ciprofloxacina $(72,6 \%)$ y sulfametoxazol trimetoprima (61,5\%); en Klebsiella oxytoca fue ampicilina (80,0\%), sulfametoxazol trimetoprima $(70,0 \%)$, ácido nalidíxico $(60,0 \%)$ y ciprofloxacina $(40,0 \%)$. Mientras que en Klebsiella pneumoniae se halló una resistencia del $(100 \%)$ para ampicilina y cefalotina, amoxicilina y ácido clavulánico $(66,7 \%)$, ciprofloxacina $(55,6 \%)$, ácido nalidíxico $(44,4 \%)$, meropenem e imipenem $(11,1 \%)$. Conclusiones. La E. coli continúa siendo el microorganismo más frecuente en ITU. El tratamiento empírico de ITU debería incluir amikacina, nitrofurantoina y piperacilina tazobactam.
\end{abstract}

Palabras clave: Prevalencia; infecciones del tracto urinario; pruebas de sensibilidad microbiana; enterobacteriaceae; resistencia a fármacos, bacteriana

\begin{abstract}
Urinary Tract Infections (UTI) are one of the main reasons for consultation in the primary care setting, due to the increase in antibacterial resistance. Objective. To characterize the prevalence of urinary tract infection and the in vitro antimicrobial susceptibility profile of Enterobacteriaceae in patients in the province of Santa Elena - Ecuador. Method. This was a descriptive research of documentary design. The population was 827 urine culture records, collected from the database of the microbiology laboratory of the Centro de Especialidades IESS La Libertad, in the period from August 2019 to March 2020. The data were processed using descriptive statistics, frequency analysis and chi-square. Results. From this study indicate that the prevalence of UTI was $22.1 \%$; the main etiological agents were: E. coli (76.0\%), Klebsiella oxytoca (6.5\%), Klebsiella pneumoniae $(5.8 \%)$ and Proteus mirabilis $(3.9 \%)$. UTI and E. coli infection were statistically higher in women and older adults. The highest frequency of E. coli resistance was for nalidixic acid $(81.2 \%)$, ampicillin (79.9\%), ciprofloxacin $(72.6 \%)$ and trimethoprim sulfamethoxazole $(61.5 \%)$; in Klebsiella oxytoca it was ampicillin $(80.0 \%)$, trimethoprim sulfamethoxazole $(70.0 \%)$, nalidixic acid $(60.0 \%)$ and ciprofloxacin $(40.0 \%)$. While in Klebsiella pneumoniae, $100 \%$ resistance was found for ampicillin and cephalothin, amoxicillin and clavulanic acid (66.7\%), ciprofloxacin (55.6\%), nalidixic acid (44.4\%), meropenem and imipenem (11.1\%). Conclusions. E. coli continues to be the most frequent microorganism in UTI. Empirical treatment of UTI should include amikacin, nitrofurantoin and piperacillin tazobactam.
\end{abstract}

Key words: Prevalence; urinary tract infections; microbial susceptibility testing; enterobacteriaceae; drug resistance, bacterial
MC: Tecnóloga Médica en Laboratorio Clínico, Universidad Estatal de Guayaquil. Licenciada en Laboratorio Clínico, Universidad Estatal de Guayaquil. Postgradista Universidad Católica de Cuenca. Maestría en Diagnóstico de Laboratorio y Molecular, Universidad Católica de Cuenca, Ecuador.

JG: Químico Farmaceuta, Universidad Católica de Cuenca. Master en Bacteriología y Micología, Universidad de la Habana. Instituto de Medicina Tropical Pedro Kourí. Coordinador Académico de la Maestría en Diagnóstico de Laboratorio Clínico y Molecular, UCACUE. Tutor de prácticas de Biología Biologia Molecular y Bacteriolog Universidad Católica de Cuenca, Ecuador. 
MC: Tecnóloga Médica en Laboratorio Clínico, Universidad Estatal de Guayaquil. Licenciada en Laboratorio Clínico, Universidad Estatal de Guayaquil. Postgradista Universidad Católica de Cuenca. Maestría en Diagnóstico de Laboratorio y Molecular, Universidad Católica de Cuenca, Ecuador.

JG: Químico Farmaceuta, Universidad Católica de Cuenca. Master en Bacteriología y Micología, Universidad de la Habana. Instituto de Medicina Tropical Pedro Kourí Coordinador Académico de la Maestría en Diagnóstico de Laboratorio Clínico y Molecular, UCACUE Tutor de prácticas de Biología Molecular y dacteriologí.

\section{RESUMO}

As infecções do trato urinário (IU) são um dos principais motivos de consulta no âmbito dos cuidados primários, devido ao aumento da resistência antibacteriana. Objetivo. Caracterizar a prevalência da infecção do trato urinário e o perfil de suscetibilidade antimicrobiana in vitro de Enterobacteriaceae em pacientes da província de Santa Elena - Equador. Método. Esta foi uma pesquisa descritiva do projeto documental. A população era de 827 registros de cultura de urina, coletados do banco de dados do laboratório de microbiologia do Centro de Especialidades IESS La Libertad, no período de agosto de 2019 a março de 2020. Os dados foram processados utilizando estatísticas descritivas, análise de freqüência e qui-quadrado. Resultados. Deste estudo indicam que a prevalência de UTI foi de 22,1\%; os principais agentes etiológicos foram: E. coli $(76,0 \%)$, Klebsiella oxytoca $(6,5 \%)$, Klebsiella pneumoniae $(5,8 \%)$ e Proteus mirabilis $(3,9 \%)$. A infecção por UTI e E. coli foi estatisticamente maior nas mulheres e nos adultos mais velhos. A maior freqüência de resistência do E. coli foi para o ácido nalidíxico $(81,2 \%)$, ampicilina $(79,9 \%)$, ciprofloxacina $(72,6 \%)$ e trimetoprim sulfametoxazol (61,5\%); em Klebsiella oxytoca era ampicilina $(80,0 \%)$, trimetoprim sulfametoxazol $(70,0 \%)$, ácido nalidíxico $(60,0 \%)$ e ciprofloxacina $(40,0 \%)$. Enquanto em Klebsiella pneumoniae, foi encontrada $100 \%$ de resistência para ampicilina e cefalotina, amoxicilina e ácido clavulânico (66,7\%), ciprofloxacina $(55,6 \%)$, ácido nalidíxico $(44,4 \%)$, meropenem e imipenem $(11,1 \%)$. Conclusões. A E. coli continua sendo o microorganismo mais freqüente na UTI. 0 tratamento empírico da UTI deve incluir amikacina, nitrofurantoína e piperacilina tazobactam.

Palavras-chave: Prevalência; infecções do trato urinário; testes de suscetibilidade microbiana; enterobactérias; resistência a drogas, bacteriana

\section{INTRODUCCIÓN}

$\mathrm{L}$ a infección del tracto urinario (ITU), es definida como el crecimiento de microorganismos patógenos en orina recogida de forma estéril con o sin presencia de síntomas clínicos compatibles (1). Este tipo de infecciones ocupan el segundo lugar entre los procesos infecciosos, siendo la principal causa de bacteriemia y sepsis en adultos mayores (2-4). Las ITU, son ocasionadas por una amplia variedad de patógenos, con mayor frecuencia se aíslan bacilos gram negativos pertenecientes a la familia Enterobacteriaceae (5).

La morbilidad por infecciones del tracto urinario es alta, del total de infecciones reportadas a nivel mundial más del $30 \%$ son debidas a ITU, de las cuales el uropatógeno más frecuente es la Escherichia coli (E. coli). La prescripción empírica de antimicrobianos en las ITU es una práctica común; por consiguiente la resistencia bacteriana a antimicrobianos se ha incrementado a nivel global, con la consecuente disminución de la efectividad del tratamiento empírico. Uno de los mecanismos de multiresistencia de mayor impacto clínico en bacterias como E. coli es la producción de $\beta$-lactamasas de espectro extendido (BLEE) (2-4).

La Organización Mundial de la Salud (OMS) en el "Reporte global sobre la vigilancia de la resistencia antimicrobiana" del 2014, reveló porcentajes elevados de resistencia a las cefalosporinas de tercera generación principalmente en $E$. coli y Klebsiella pneumoniae (K. pneumoniae), tanto en infecciones nosocomiales como en infecciones adquiridas en la comunidad. En este contexto, la OMS estima que en el año 2050 el continuo incremento de la resistencia antimicrobiana producirá un impacto clínico-económico relevante, estimándose diez millones de muertes 
anuales y la reducción del producto interno bruto mundial entre $2 \%$ y $3,5 \%(6)$.

América Latina, no está exenta frente a esta problemática. En Colombia, la mayor frecuencia de resistencia en E. coli fue para ampicilina (61\%), seguido de ácido nalidíxico y trimetoprim-sulfametoxazol (48\%). En Klebsiella spp., la frecuencia de resistencia fue menor con el $23 \%$ para trimetoprim-sulfametoxazol,

$22 \%$ ampicilina-sulbactam, $19 \%$ cefalotina, $19 \%$ nitrofurantoina y $15 \%$ ciprofloxacina (7).

Asimismo en Brasil, el antibiótico con mayor prevalencia de resistencia bacteriana fue sulfametoxazol-trimetoprim en promedio $46,9 \%$, seguido de cefalotina $46,7 \%$, ácido nalidíxico 27,6\% y nitrofurantoina 22,3\%(8).

En Ecuador, un estudio realizado en la ciudad Quito determinó que $E$. coli presenta tasas de resistencia a ampicilina $>50 \%$, trimetoprimsulfametoxazol $>20 \%$, ciprofloxacina $56.8 \%$, gentamicina $19.4 \%$ y amikacina $3.6 \%(9)$.

La resistencia antimicrobiana cobra cada día más relevancia dado que es un serio problema de salud en todo el mundo y constituye un reto mayor para el futuro (10).Esto implica que las consecuencias negativas se ven expresadas en términos de morbilidad, mortalidad y gastos sanitarios derivados de la atención médica que podrían amenazar la sostenibilidad de los sistemas de salud (2).

El uso excesivo de antibióticos promueve la generación de cepas resistentes, limitando las opciones terapéuticas disponibles en el campo clínico. La aparición de cepas resistentes frente a varios grupos de antimicrobianos está relacionada con el uso inapropiado de antimicrobianos, la automedicación y el incumplimiento terapéutico $(2,11)$.

La presente investigación resulta novedosa ya que en la provincia de Santa Elena en Ecuador se desconoce cuál es la prevalencia de infección urinaria adquirida en la comunidad, así como también el perfil de susceptibilidad antimicrobiana.

Este tabajo tiene como objetivo caracterizar la infección urinaria adquirida en la comunidad y su respectivo perfil de susceptibilidad antimicrobiana en Enterobacterias según su prevalencia en los pacientes de la provincia de Santa Elena-Ecuador, comprendido en el período desde agosto del 2019 hasta marzo de 2020 .

\section{MATERIALES Y MÉTODOS}

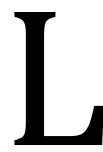

a presente investigación fue descriptiva de diseño documental, la población de estudio estuvo conformada por 827 registros en la base de datos que se realizaron urocultivos en el laboratorio del Centro de Especialidades IESS La Libertad, desde agosto 2019 hasta marzo de 2020. Se utilizó un muestreo de tipo cobertura total, los datos fueron obtenidos de fuentes secundarias ingresados en los registros del departamento de Bacteriología.

Criterios de inclusión: Registros de pacientes que se realizaron urocultivos, registros de pacientes que fueron reportados sin crecimiento bacteriano, registros clínicos diagnosticados como productores de ITU. Registros de enterobacterias que presenten resistencia al menos a un antimicrobiano.

Criterios de exclusión: Registros clínicos que no tengan información completa, registros de urocultivos que 
fueron reportados como muestras contaminadas, urocultivos que sean positivo para cocos grampositivo.

Para el análisis estadístico se generó una base de datos en el programa SPSS 21.0. Los datos fueron procesados mediante estadística descriptiva, análisis de frecuencia y chi cuadrado.

Para la presentación de los resultados se utilizó tablas de simple y doble entrada, tablas cruzadas y se representaron los gráficos mediante el empleo de diagrama de barras y diagrama de sectores.

\section{Aspectos Éticos}

Esta investigación fue fundamentada en los principios éticos establecidos en la Declaración de Helsinki Adendum de Taiwán 2016, se dio protección y privacidad a los datos de los pacientes de los cuales se obtuvieron los registros clínicos objetos de estudio; fueron manejados con estricta confidencialidad; no se vulneró el derecho de ningún paciente. Se protegió la base de datos con clave de seguridad restringiendo su acceso; la información no se utilizó con otros fines que no sean de carácter investigativo, no tuvo manejo público y no se harán nuevas investigaciones, respetando para lo que fueron tomadas, que fue el diagnóstico clínico.

\section{RESULTADOS}

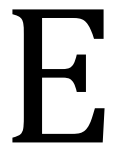
n este estudio se evidenció que la prevalencia de las ITU fue del $22,1 \%$ con respecto al total de urocultivos realizados en los años mencionados (Ver Tabla 1).

Tabla 1. Prevalencia de las infecciones del tracto urinario en el Centro de Especialidades IESS La Libertad. Santa Elena- Ecuador, en el período de agosto 2019 - marzo 2020.

\begin{tabular}{clcccc}
\hline & \multicolumn{3}{c}{ PATÓGENO } & & \\
& & Frecuencia & Porcentaje & $\begin{array}{c}\text { Porcentaje } \\
\text { válido }\end{array}$ & $\begin{array}{c}\text { Porcentaje } \\
\text { acumulado }\end{array}$ \\
\hline \multirow{3}{*}{ Válidos } & POSITIVOS & 154 & 22,1 & 22,1 & 22,1 \\
& NEGATIVOS & 543 & 77,9 & 77,9 & 100,0 \\
& Total & 697 & 100,0 & 100,0 & \\
\hline
\end{tabular}

Según los datos obtenidos se puede observar que las infecciones de vías urinarias se presentan con mayor frecuencia en el género femenino, con un porcentaje de $72,7 \%$, seguido del masculino con un porcentaje de 27,3\%. $\left(X^{2}=31,818 ; \mathrm{p}=0,000\right)$; (ver Tabla 2 ).

Tabla 2. Distribución por género de las infecciones del tracto urinario en el Centro de Especialidades IESS La Libertad. Santa Elena- Ecuador, en el período de agosto 2019 marzo 2020.

\begin{tabular}{cccccc}
\hline & \multicolumn{2}{c}{ GÉNERO } & Porcentaje & $\begin{array}{c}\text { Porcentaje } \\
\text { acumulado }\end{array}$ \\
\hline Válidos & FEMENINO & 112 & 72,7 & 72,7 & 72,7 \\
& MASCULINO & 42 & 27,3 & 27,3 & 100,0 \\
& Total & 154 & 100,0 & 100,0 & \\
\hline
\end{tabular}


En relación al servicio hospitalario la gran parte de urocultivos positivos vienen del servicio de urología $(36,4 \%)$, seguido de medicina general $(15,6 \%)$ y medicina interna $(13,6 \%) \cdot\left(X^{2}=104,49\right.$ y p=0,000); (ver Gráfico 1).

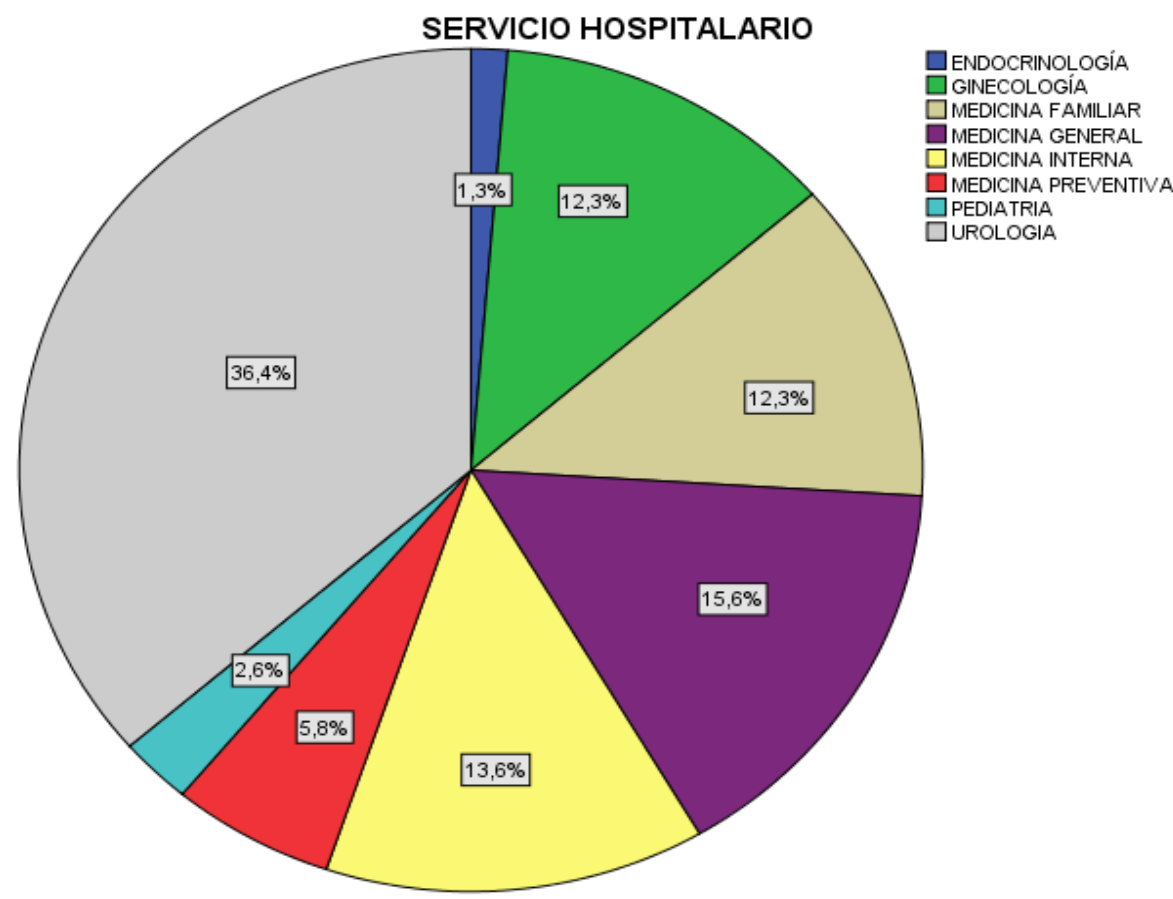

Gráfico 1. Porcentaje de infecciones del tracto urinario según servicio hospitalario en pacientes Centro de Especialidades IESS La Libertad. Santa Elena-Ecuador, en el período de agosto 2019-marzo 2020.

Al comparar la prevalencia de ITU y los uropatógenos según el grupo etario, los adultos mayores obtuvieron un porcentaje de $46,8 \%$.

Los principales agentes etiológicos fueron $E$. coli con un valor de 76,0 \%, seguido de Klebsiella oxytoca con 6,5\%,
Klebsiella pneumoniae 5,8\% y Proteus mirabilis con 3,9\%. Llama la atención que Pseudomona aeruginosa tenga un porcentaje de 1,3\%, siendo un uropatógeno a nivel intrahospitalario y no en la comunidad. (Ver Gráfico 2). 


\section{UROPATÓGENOS}

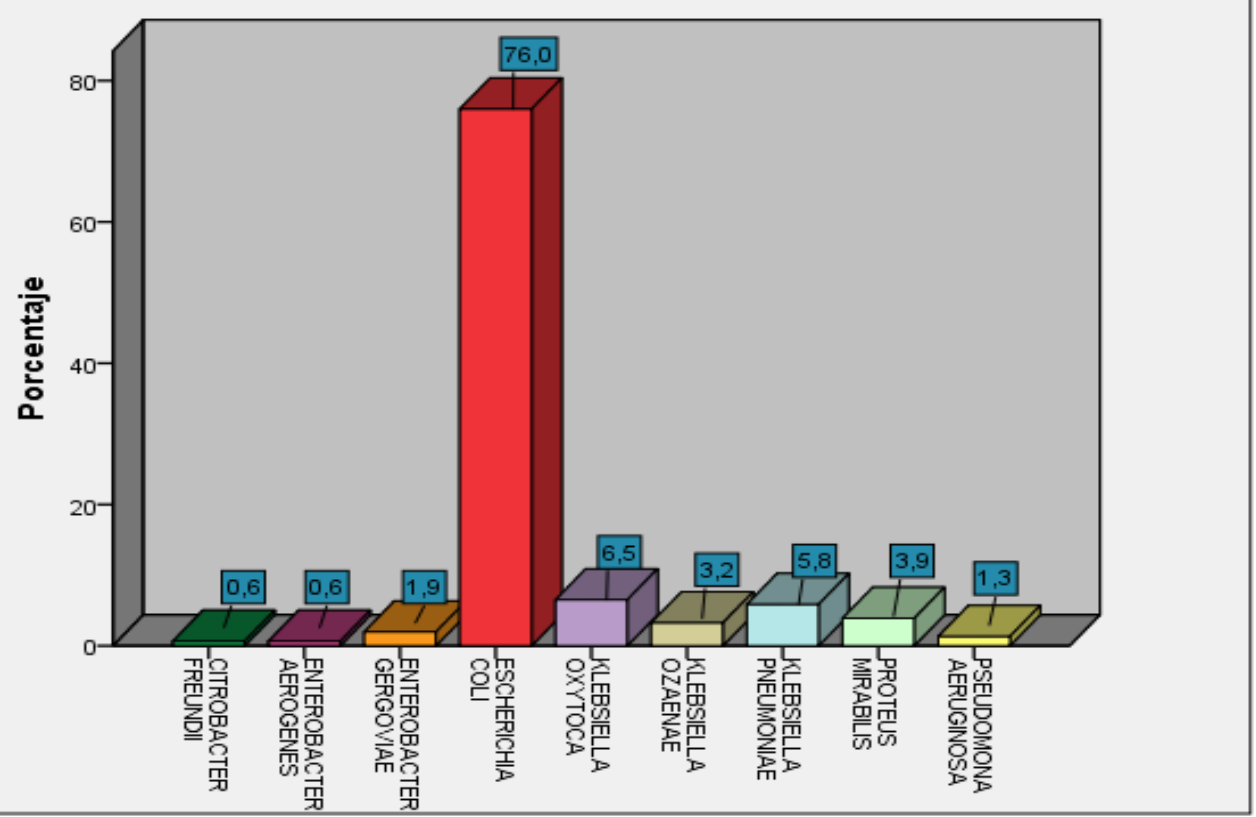

Gráfico 2. Porcentaje de uropatógenos de infecciones del tracto urinario en pacientes Centro de Especialidades IESS La Libertad. Santa Elena- Ecuador, en el período de agosto 2019-marzo 2020.

La prevalencia de BLEE es del 18,8 \%, el uropatógeno con más alto porcentaje en relación a este mecanismo de resistencia es la Klebsiella ozaenae con 40,0\%.(ver Tabla 3).

Tabla 3. Mecanismo de resistencia BLEE de las infecciones del tracto urinario en el Centro de Especialidades IESS La Libertad. Santa Elena-Ecuador, en el período de agosto 2019marzo 2020.

\begin{tabular}{|c|c|c|c|c|c|}
\hline \multicolumn{6}{|c|}{ MECANISMO DE RESISTENCIA } \\
\hline & & & NEGATIVO & BLEE & Total \\
\hline \multirow{10}{*}{ PATÓGENO } & & Recuento & 1 & 0 & 1 \\
\hline & Citrobacter freundii & $\begin{array}{l}\text { \% dentro de } \\
\text { PATÓGENO }\end{array}$ & $100,0 \%$ & $0,0 \%$ & $100,0 \%$ \\
\hline & & Recuento & 1 & 0 & 1 \\
\hline & Enterobacter aerogenes & $\begin{array}{l}\text { \% dentro de } \\
\text { PATÓGENO }\end{array}$ & $100,0 \%$ & $0,0 \%$ & $100,0 \%$ \\
\hline & & Recuento & 3 & 0 & 3 \\
\hline & Enterobacter gergoviae & $\begin{array}{l}\% \text { dentro de } \\
\text { PATÓGENO }\end{array}$ & $100,0 \%$ & $0,0 \%$ & $100,0 \%$ \\
\hline & & Recuento & 92 & 25 & 117 \\
\hline & Escherichia coli & $\begin{array}{l}\text { \% dentro de } \\
\text { PATÓGENO }\end{array}$ & $78,6 \%$ & $21,4 \%$ & $100,0 \%$ \\
\hline & & Recuento & 9 & 1 & 10 \\
\hline & Klebsiella oxytoca & $\begin{array}{l}\text { \% dentro de } \\
\text { PATÓGENO }\end{array}$ & $90,0 \%$ & $10,0 \%$ & $100,0 \%$ \\
\hline
\end{tabular}




\begin{tabular}{|c|c|c|c|c|c|}
\hline \multicolumn{6}{|c|}{ MECANISMO DE RESISTENCIA } \\
\hline & & & NEGATIVO & BLEE & Total \\
\hline & \multirow[t]{3}{*}{ Klebsiella ozaenae } & Recuento & 3 & 2 & 5 \\
\hline & & $\begin{array}{l}\% \text { dentro de } \\
\text { PATÓGENO }\end{array}$ & $60,0 \%$ & $40,0 \%$ & $100,0 \%$ \\
\hline & & Recuento & 9 & 0 & 9 \\
\hline & \multirow[t]{2}{*}{ Klebsiella pneumoniae } & $\begin{array}{l}\% \text { dentro de } \\
\text { PATÓGENO }\end{array}$ & $100,0 \%$ & $0,0 \%$ & $100,0 \%$ \\
\hline & & Recuento & 5 & 1 & 6 \\
\hline & \multirow[t]{2}{*}{ Proteus mirabilis } & $\begin{array}{l}\text { \% dentro de } \\
\text { PATÓGENO }\end{array}$ & $83,3 \%$ & $16,7 \%$ & $100,0 \%$ \\
\hline & & Recuento & 2 & 0 & 2 \\
\hline & \multirow[t]{3}{*}{ Pseudomona aeruginosa } & $\begin{array}{l}\text { \% dentro de } \\
\text { PATÓGENO }\end{array}$ & $100,0 \%$ & $0,0 \%$ & $100,0 \%$ \\
\hline & & Recuento & 125 & 29 & 154 \\
\hline Total & & $\begin{array}{l}\text { \% dentro de } \\
\text { PATÓGENO }\end{array}$ & $81,2 \%$ & $18,8 \%$ & $100,0 \%$ \\
\hline
\end{tabular}

En cuanto, al perfil de susceptibilidad antimicrobiana se mostró los tres microorganismos más prevalentes en la población estudiada. El mayor porcentaje de resistencia para $E$. coli fue ácido nalidíxico $81,2 \%$, ampicilina $79,9 \%$, ciprofloxacina $72,6 \%$, sulfametoxazol trimetoprima $61,5 \%$, cefalotina $60,7 \%$.

Para Klebsiella oxytoca fue ampicilina $80,0 \%$, sulfametoxazol trimetoprima
$70,0 \%$, ácido nalidíxico 60,0 \%, ciprofloxacina $40,0 \%$, cefalotina $40,0 \%$.

En Klebsiella pneumoniae se halló una resistencia del 100\% para ampicilina y cefalotina, amoxicilina y ácido clavulánico $66,7 \%$, ciprofloxacina 55,6\%, ácido nalidíxico 44,4\%, meropenem e imipenem 11,1\% (ver Tabla 4).

Tabla 4. Perfil de susceptibilidad antimicrobiana de los principales uropatógenos hallados en las infecciones del tracto urinario en el Centro de Especialidades IESS La Libertad. Santa Elena- Ecuador, en el período de agosto 2019-marzo 2020.

\begin{tabular}{|c|c|c|c|c|}
\hline $\begin{array}{l}\text { SUSCEPTIBILIDAD } \\
\text { ANTIMICROBIANA }\end{array}$ & & E. coli & K. oxytoca & K. pneumoniae \\
\hline \multirow{3}{*}{$\begin{array}{c}\text { ÁCIDO } \\
\text { NALIDÍXICo }\end{array}$} & S & $13,7 \%(16)$ & $10,0 \%(1)$ & $44,4 \%(4)$ \\
\hline & I & $5,1 \%(6)$ & $30,0 \%(3)$ & $11,1 \%(1)$ \\
\hline & $\mathrm{R}$ & $81,2 \%(95)$ & $60,0 \%(6)$ & $44,4 \%(4)$ \\
\hline \multirow{3}{*}{ AMPICILINA } & S & $16,2 \%(19)$ & $20,0 \%(2)$ & $0,0 \%(0)$ \\
\hline & I & $4,3 \%(5)$ & $0,0 \%(0)$ & $0,0 \%(0)$ \\
\hline & $\mathrm{R}$ & $79,5 \%$ (93) & $80,0 \%$ (8) & $100,0 \%(9)$ \\
\hline \multirow{3}{*}{ CIPROFLOXACINA } & $\mathrm{S}$ & $19,7 \%(23)$ & $40,0 \%(4)$ & $44,4 \%(4)$ \\
\hline & I & $7,7 \%$ (9) & $20,0 \%$ (2) & $0,0 \%(0)$ \\
\hline & $\mathrm{R}$ & $72,6 \%(85)$ & $40,0 \%(4)$ & $55,6 \%(5)$ \\
\hline \multirow{3}{*}{$\begin{array}{l}\text { TRIMETOPRIMA + } \\
\text { SULFAMETOXAZOL }\end{array}$} & $\mathrm{S}$ & $37,6 \%(44)$ & $30,0 \%(3)$ & $66,7 \%(6)$ \\
\hline & I & $0,9 \%(1)$ & $0,0 \%(0)$ & $0,0 \%(0)$ \\
\hline & $\mathrm{R}$ & $61,5 \%(72)$ & $70,0 \%(7)$ & $33,3 \%(3)$ \\
\hline
\end{tabular}




\begin{tabular}{|c|c|c|c|c|}
\hline $\begin{array}{l}\text { SUSCEPTIBILIDAD } \\
\text { ANTIMICROBIANA }\end{array}$ & & E. coli & K. oxytoca & K. pneumoniae \\
\hline \multirow{3}{*}{ CEFALOTINA } & S & $39,3 \%(46)$ & $60,0 \%(6)$ & $33,3 \%(3)$ \\
\hline & I & $60,7 \%(71)$ & $40,0 \%(4)$ & $66,7 \%(6)$ \\
\hline & $\mathrm{R}$ & $100,0 \%$ (117) & $100,0 \%(10)$ & $100,0 \%(9)$ \\
\hline \multirow{4}{*}{$\begin{array}{l}\text { AMOXICILINA+ } \\
\text { ACIDO } \\
\text { CLAVULÁNICO }\end{array}$} & S & $41,0 \%(48)$ & $40,0 \%(4)$ & $33,3 \%(3)$ \\
\hline & I & $20,5 \%(24)$ & $40,0 \%(4)$ & $0,0 \%(0)$ \\
\hline & $\mathrm{R}$ & $38,5 \%(45)$ & $20,0 \%(2)$ & $66,7 \%(6)$ \\
\hline & S & $65,8 \%(77)$ & $90,0 \%(9)$ & $66,7 \%(6)$ \\
\hline \multirow[t]{3}{*}{ CEFEPIME } & I & $34,2 \%(40)$ & $10,0 \%(1)$ & $33,3 \%(3)$ \\
\hline & $\mathrm{R}$ & $100,0 \%(117)$ & $100,0 \%(10)$ & $100,0 \%(9)$ \\
\hline & $\mathrm{S}$ & $62,4 \%(73)$ & $80,0 \%(8)$ & $66,7 \%(6)$ \\
\hline \multirow[t]{3}{*}{ CEFOTAXIMA } & I & $3,4 \%(4)$ & $10,0 \%(1)$ & $0,0 \%(0)$ \\
\hline & $\mathrm{R}$ & $34,0 \%(40)$ & $10,0 \%(1)$ & $33,3 \%(3)$ \\
\hline & S & $64,1 \%(75)$ & $80,0 \%(8)$ & $66,7 \%(6)$ \\
\hline \multirow[t]{3}{*}{ CEFTRIAXONA } & I & $1,7 \%(2)$ & $10,0 \%(1)$ & $0,0 \%(0)$ \\
\hline & $\mathrm{R}$ & $34,2 \%(40)$ & $10,0 \%(1)$ & $33,3 \%(3)$ \\
\hline & S & $65,8 \%(77)$ & $90,0 \%(9)$ & $77,8 \%(7)$ \\
\hline \multirow[t]{3}{*}{ GENTAMICINA } & I & $34,2 \%(40)$ & $10,0 \%(1)$ & $22,2 \%(2)$ \\
\hline & $\mathrm{R}$ & $100,0 \%$ (117) & $100,0 \%(10)$ & $100,0 \%(9)$ \\
\hline & S & $66,7 \%(78)$ & $70,0 \%(7)$ & $66,7 \%(6)$ \\
\hline \multirow{3}{*}{ CEFTAZIDIMA } & I & $6,8 \%(8)$ & $10,0 \%(1)$ & $0,0 \%(0)$ \\
\hline & $\mathrm{R}$ & $26,5 \%(31)$ & $20,0 \%(2)$ & $0,0 \%(0)$ \\
\hline & S & $86,3 \%(101)$ & $90,0 \%(9)$ & $100,0 \%(9)$ \\
\hline \multirow[t]{3}{*}{ AMIKACINA } & I & $4,3 \%(5)$ & $0,0 \%(0)$ & $0,0 \%(0)$ \\
\hline & $\mathrm{R}$ & $9,4 \%(11)$ & $10,0 \%(1)$ & $0,0 \%(0)$ \\
\hline & $\mathrm{S}$ & $91,5 \%(107)$ & $80,0 \%(8)$ & $55,6 \%(5)$ \\
\hline \multirow[t]{3}{*}{ NITROFURANTOINA } & I & $3,4 \%(4)$ & $0,0 \%(0)$ & $0,0 \%(0)$ \\
\hline & $\mathrm{R}$ & $5,1 \%(6)$ & $20,0 \%(2)$ & $44,4 \%(4)$ \\
\hline & S & $82,1 \%(96)$ & $100,0 \%(10)$ & $88,9 \%(8)$ \\
\hline \multirow{3}{*}{$\begin{array}{l}\text { PIPERACILINA } \\
\text { +TAZOBACTAM }\end{array}$} & I & $13,7 \%(16)$ & $0,0 \%(0)$ & $0,0 \%(0)$ \\
\hline & $\mathrm{R}$ & $4,3 \%(5)$ & $0,0 \%(0)$ & $11,1 \%(1)$ \\
\hline & S & $96,6 \%(113)$ & $100,0 \%(10)$ & $88,9 \%(8)$ \\
\hline \multirow[t]{3}{*}{ IMIPENEM } & I & $1,7 \%(2)$ & $0,0 \%(0)$ & $0,0 \%(0)$ \\
\hline & $\mathrm{R}$ & $1,7 \%(2)$ & $0,0 \%(0)$ & $11,1 \%(1)$ \\
\hline & S & $99,1 \%(116)$ & $100,0 \%(10)$ & $88,9 \%(8)$ \\
\hline \multirow[t]{2}{*}{ MEROPENEM } & I & $0,9 \%(1)$ & $0,0 \%(0)$ & $0,0 \%(0)$ \\
\hline & $\mathrm{R}$ & $0,0 \%(0)$ & $0,0 \%(0)$ & $11,1 \%(1)$ \\
\hline
\end{tabular}




\section{DISCUSIÓN}

$\mathrm{L}$ as ITU son una de las enfermedades infecciosas diagnosticadas con mayor frecuencia en la práctica clínica $(2,4)$.

La mayor prevalencia de ITU se encontró en los adultos mayores $(\geq 65$ años), este hallazgo concuerda con el estudio realizado por Smithson et al., en Europa. Posiblemente podría deberse a que la mayoría de adultos mayores tienen enfermedades comórbidas, fragilidad y un sistema inmunológico debilitado $(12,13)$.

La presencia de ITU mayoritariamente en el sexo femenino concuerda con lo reportado hasta la actualidad, ya que ciertas características anatómicas (vagina, uretra y ano) de las mujeres predisponen a las ITU (14). Además, se asocian factores de riesgo como: inicio de la actividad sexual, embarazo, uso de condón o diafragma con espermicida, tampón vaginal, terapia antimicrobiana previa, antecedente de primer episodio de ITU antes de los 15 años de edad, madre con antecedente de ITU recurrente y nueva pareja sexual en el último año $(15,16)$.

De manera similar a lo reportado por Gordillo en Quito - Ecuador (9), E. coli fue el uropatógeno más prevalente en ITU. En consonancia a estudios de otros países como lo evidenció Orrego en Colombia (7) y Marrero en Cuba (17), lo que supone que E. coli es el principal patógeno relacionado con el desarrollo de ITU a nivel mundial. Los hallazgos de este estudio sobre Pseudomona aeruginosa difiere con los de Guevara (18). Esta discrepancia podría atribuirse a que Pseudomonas es un uropatógeno común en infecciones del tracto urinario a nivel intrahospitalario, sobre todo en pacientes sometidos a manipulación urológica, uropatía obstructiva o que han recibido antibióticos de amplio espectro (19). Se puede suponer que Pseudomonas aeruginosa se encuentra en la comunidad debido a que en el servicio de urología acuden pacientes con sondas vesicales permanentes o con cistectomía.

E. coli y K. pneumoniae se encuentran entre los microorganismos productores de BLEE más prevalentes y son causa frecuente de infecciones hospitalarias y comunitarias $(2,4,20)$. En Santa ElenaEcuador Klebsiella ozaenae y E. coli son los principales uropatógenos productores de BLEE, a diferencia del estudio de Navarro y cols. En México (20). La producción de BLEE se relaciona con fallas terapéuticas y es un serio problema para el control de infecciones mostrando un alto nivel de resistencia a los antimicrobianos comúnmente y disminuyendo las opciones terapéuticas para tratamientos empíricos (4).

Con respecto a los patrones de resistencia a los antimicrobianos de uso común en el tratamiento de ITU en este estudio, se encontró que para el manejo de las infecciones causadas por E. coli el ácido nalidíxico y ampicilina no son una buena opción terapéutica, ambos antibióticos presentaron las mayores tasas de resistencia, seguido de ciprofloxacina y sulfametoxazol trimetoprima. Similar a lo hallado en otros estudios como el de Caicedo en Colombia (21) y Marrero en Cuba (17). Además, en este estudio se observó una elevada resistencia a ciprofloxacina, hecho que difiere de lo encontrado en el estudio de Miranda (2) y concuerda con el de Montañez (22).

Otro hallazgo importante fue la resistencia a los carbapenémicos en $K$. pneumoniae. La OMS en el año 2014, informó que las cepas de $K$. pneumoniae 
resistentes a carbapenémicos se han diseminado mundialmente debido a la transmisión horizontal de genes (plásmidos, transposones e integrones) y que el principal mecanismo de resistencia es la producción de enzimas carbapenemasas entre las que destacan las KPC (Klebsiella pneumoniae productoras de carbapenemasas) (23). Una explicación tentativa para estos resultados podría ser que la resistencia de este uropatógeno está relacionada con la producción de KPC (24).

Con ello se evidencia que la resistencia varía entre poblaciones, por lo que se recomienda disponer de perfiles clínico epidemiológicos locales y se concluye que para los pacientes estudiados estos antimicrobianos no tienen una buena efectividad.

\section{CONCLUSIONES}

$\mathrm{D}$ entro de las Enterobacterias, la Escherichia coli es el uropatógeno más frecuente en la población objeto de estudio, causante de infecciones urinarias en la comunidad, con mayor incidencia en el género femenino. Para el tratamiento empírico la tasa de resistencia microbiana no debe ser superior al 20\% (25). De este modo, según los resultados microbiológicos, se indica que los antibióticos que podrían ser utilizados empíricamente para el tratamiento de la ITU son amikacina, nitrofurantoina, piperacilina tazobactam. Sin embargo, sería de gran beneficio que se realicen urocultivos previo a prescripción médica de antibióticos.

La diversidad de uropatógenos aislados y la variabilidad de perfiles de resistencia antimicrobiana evidencian la necesidad de fomentar investigaciones locales que permitan el monitoreo rutinario de patrones de resistencia para promover la prescripción apropiada de antibióticos orientada a la vigilancia epidemiológica de medicamentos, acorde a cada población de estudio.

\section{REFERENCIAS BIBLIOGRÁFICAS}

1. Echevarría J, Sarmiento E, Osores F. Infección del tracto urinario y manejo antibiótico. Acta Médica Peru [Internet]. enero de 2006;23(1):26-31. Disponible en: http://www.scielo.org.pe/scielo.php?script=s ci_abstract\&pid=S1728-

59172006000100006\&lng=es\&nrm=iso\&thn $\mathrm{g}=\mathrm{es}$

2. Miranda J, Pinto J, Faustino M, SánchezJacinto B, Ramirez F. Resistencia antimicrobiana de uropatógenos en adultos mayores de una clínica privada de Lima, Perú. Rev Peru MedExp Salud Pública [Internet]. marzo de 2019 [citado 29 de abril de 2020];36(1):87-92. Disponible en: https://rpmesp.ins.gob.pe/index.php/rpmes $\mathrm{p} /$ article/view/3765

3. Guajardo C, González P, Ayala J. Resistencia antimicrobiana en la infección urinaria por Escherichia coli adquirida en la comunidad: ¿Cuál antibiótico voy a usar? Salud Pública México [Internet]. abril de 2009 [citado 29 de abril de 2020];51:155-9. Disponible en: https://scielosp.org/article/ spm/2009.v51n2/155-159/es/

4. Galindo M. Molecular characterization and antimicrobialsusceptibilitypattern of extended-spectrum $\beta$-lactamase-producing Escherichia coli as cause of communityacquiredurinarytractinfection.

RevChilInfectol [Internet]. 2018 [citado 28 de abril de 2020];35(1):29-35. Disponible en: https://scielo.conicyt.cl/scielo.php?script=sci _abstract\&pid=S0716-101820180001000 29\&lng=en\&nrm=iso\&tlng=en

5. Aguinaga A, Gil A, Mazón A, Alvaro A, García J, Navascués A, etal. Infecciones del tracto urinario. Estudio de sensibilidad antimicrobiana en Navarra. AnSistSanitNavar [Internet]. abril de 2018 [citado 19 de mayo de 2020];41(1):17-26. Disponible en: 
http://scielo.isciii.es/scielo.php?script=sci_ab stract\&pid=S1137-66272018000100017\& lng=es\&nrm=iso\&tlng=es

6. Yagui M. Resistencia antimicrobiana: nuevo enfoque y oportunidad. Rev Peru MedExp Salud Pública [Internet]. abril de 2018 [citado 29 de abril de 2020];35(1):7-8. Disponible en: https://rpmesp.ins.gob.pe/ index.php/rpmesp/article/view/3594

7. Orrego C, Henao C, Cardona J. Prevalencia de infección urinaria, uropatógenos y perfil de susceptibilidad antimicrobiana. Acta Medica Colomb [Internet]. octubre de 2014 [citado 21 de diciembre de 2020];39(4):352-8. Disponible en: http://www.scielo.org.co/scielo.php? script=sci_abstract\&pid=S0120-244820 1400040 0008\&lng=en\&nrm=iso\&tlng=es

8. Koch C, Ribeiro J, Schnor O, Zimmermann B, Müller FM, D’ Agostin J, et al. Resistência antimicrobiana dos uropatógenos em pacientes ambulatoriais, 2000-2004. RevSocBrasMedTrop [Internet]. junio de 2008 [citado 10 de diciembre de 2020];41(3):277-81. Disponible en: http://www.scielo.br/scielo.php?script=sci_a bstract\&pid=S0037-868220080003000 $10 \& \operatorname{lng}=$ en\&nrm=iso\&tlng=pt

9. Gordillo F, Barrera F. Perfil de resistencia de uropatógenos en pacientes con diabetes en Quito, Ecuador, inquietante panorama. Salud Pública México [Internet]. febrero de 2018 [citado 21 de mayo de 2020];60:97-8. Disponible en: https://scielosp.org/article/spm/2018.v60n 1/97-98/

10. Valdés $S$, Ángel $M$. La resistencia microbiana en el contexto actual y la importancia del conocimiento y aplicación en la política antimicrobiana. Rev Habanera Cienc Médicas [Internet]. junio de 2017 [citado 19 de mayo de 2020];16(3):402-19. Disponible en: http://scielo.sld.cu/scielo.php ?script=sci_abstract\&pid=S1729-519X20170 00300011\&lng=es\&nrm=iso\&tlng=es

11. Alzamora M, Echevarría A, Ferraro V, Riveros M, Zambruni M, Ochoa T. Resistencia antimicrobiana de cepas comensales de Escherichia coli en niños de dos comunidades rurales peruanas. Rev Peru MedExp Salud Pública [Internet]. septiembre de 2019 [citado 29 de abril de 2020];36(3):459-63. Disponible en: https://rpmesp.ins.gob.pe/ index.php/rpmesp/article/view/4366

12. Smithson A, Ramos J, Niño E, Culla A, Pertierra U, Friscia M, et al. Characteristics of febrileurinarytractinfections in oldermaleadults. BMC Geriatr [Internet]. 29 de noviembre de 2019 [citado 25 de febrero de 2021];19. Disponible en: https://www.ncbi.nlm.nih.gov/pmc/articles/ PMC6884897/

13. Blanco M, Kou S, L. La comorbilidad y su valor para el médico generalista en Medicina Interna. Rev Habanera Cienc Médicas [Internet]. febrero de 2017 [citado 7 de marzo de 2021];16(1):12-24. Disponible en: http://scielo.sld.cu/scielo.php?script=sci_abst ract\&pid=S1729-519X2017000100004\&lng =es\&nrm=iso\&tlng=es

14. Calderón E, Casanova G, Galindo A, Gutiérrez $\mathrm{P}$, Landa $\mathrm{S}$, Moreno $\mathrm{S}$, et al. Diagnóstico y tratamiento de las infecciones en vías urinarias: un enfoque multidisciplinario para casos no complicados. Bol MédHospInfant México

[Internet]. febrero de 2013 [citado 7 de marzo de 2021];70(1):03-10. Disponible en: http://www.scielo.org.mx/scielo.php?script= sci_abstract\&pid=S1665-11462013000 100003\&lng=es\&nrm=iso\&tlng=es

15. Valdevenito J, Álvarez D. Infección urinaria recurrente en la mujer. Rev Médica Clínica Las Condes [Internet]. 1 de marzo de 2018 [citado 7 de marzo de 2021];29(2):22231. Disponible en: http://www.elsevier.es /es-revista-revista-medica-clinica-las-condes 202-articulo-infeccion-urinaria-recurrentemujer-S0716 864018300282

16. Scholes D, Hooton TM, Roberts PL, Stapleton AE, Gupta K, Stamm WE. RiskFactorsforRecurrentUrinaryTractInfectin in Young Women. J InfectDis [Internet]. 1 de octubre de 2000 [citado 7 de marzo de 2021];182(4):1177-82. Disponible en: https://doi.org/10.1086/315827 
17. Marrero J, M., Castellanos J. Infección del tracto urinario y resistencia antimicrobiana en la comunidad. Rev Cuba Med Gen Integral [Internet]. marzo de 2015 [citado 17 de mayo de 2020];31(1):78-84. Disponible en: http://scielo.sld.cu/scielo.php?script=sci_abst ract\&pid=S0864-21252015000100011\&lng= es\&nrm=iso\&tlng=es

18. Guevara P A, Machado B S, Manrique $T$ E. Infecciones urinarias adquiridas en la comunidad: epidemiología, resistencia a los antimicrobianos y opciones terapéuticas. Kasmera [Internet]. diciembre de 2011 [citado 25 de febrero de 2021];39(2):87-97. Disponible en: http://ve.scielo.org/scielo. php?script=sci_abstract\&pid=S0075-5222 2011000200002\&lng=es\&nrm=iso\&tlng=es

19. Lebeque $\mathrm{Y}$, Morris $\mathrm{H}$, Calás $\mathrm{N}$. Infecciones nosocomiales: incidencia de la Pseudomonasaeruginosa. Rev Cuba Med [Internet]. marzo de 2006 [citado 14 de marzo de 2021];45(1):0-0. Disponible en: http://scielo.sld.cu/scielo.php?script=sci_abst ract\&pid=S0034-75232006000100005\& lng=es\&nrm=iso\&tlng=es

20. Navarro M, Robles R, Garibay A, Ruiz E. Escherichia coli y Klebsiella pneumoniae comunitarias y hospitalarias productoras de $\beta$-lactamasas en hospitales de Hermosillo, Sonora. Salud Pública México [Internet]. agosto de 2011 [citado 25 de febrero de 2021];53(4):341-4. Disponible en: http://www.scielosp.org/scielo.php?script=s ci_arttext\&pid=S0036-36342011000400 009\&lng=es\&nrm=iso\&tlng=es

21. Caicedo $P$, Martínez $T$, Menéses $E$, Germán J, Imbachí I R. Etiología y resistencia bacteriana en infección de vias urinarias en el Hospital Universitario San José de Popayán, Colombia entre enero y diciembre de 2008. P S. :9.
22. Montañez R, Montenegro J, Arenas F, Vásquez R. Infección urinaria alta comunitaria por E.coli resistente a ciprofloxacino: características asociadas en pacientes de un hospital nacional en Perú. AnFacMed [Internet]. octubre de 2015 [citado 25 de febrero de 2021];76(4):385-91. Disponible en: http://www.scielo.org.pe/scielo.php ?script=sci_abstract\&pid=S1025-5583201 $5000500009 \& \operatorname{lng}=e s \& n r m=i s o \& t \operatorname{lng}=e s$

23. WHO_HSE_PED_AIP_2014.2_eng.pdf [Internet]. [citado 15 de marzo de 2021]. Disponible en: https://apps.who.int/iris/ bitstream/handle/10665/112647/WHO_HS E_PED_AIP_2014.2_eng.pdf

24. Vera A, Barría $C$, Carrasco $S$, Lima $C$, Aguayo A, Domínguez M, et al. KPC: Klebsiella pneumoniae carbapenemasa, principal carbapenemasa en enterobacterias. RevChilInfectol [Internet]. octubre de 2017 [citado 15 de marzo de 2021];34(5):476-84. Disponible en: https://scielo.conicyt.cl/scielo. php?script=sci_abstract\&pid=S0716-1018 20170005004 76\&lng=es\&nrm=iso\&tlng=es

25. Castro R, Barreto A, Guzmán H, Ortega $\mathrm{R}$, Benítez L. Patrones de resistencia antimicrobiana en uropatógenos gramnegativos aislados de pacientes ambulatorios y hospitalizados Cartagena, 2005-2008. Rev Salud Pública [Internet]. diciembre de 2010 [citado 25 de febrero de 2021];12:1010-9. Disponible en: https://www.scielosp.org/article/rsap/2010. v12n6/1010-1019/

Conflicto de interese: Los autores declaran que no existe conflicto de intereses para la publicación del presente artículo.

Financiamiento: Autofinanciamiento

Agradecimiento: No declaran 